\title{
Comparing standardized coefficients in structural equation modeling: a model reparameterization approach
}

\author{
Joyce L. Y. Kwan • Wai Chan
}

Published online: 22 April 2011

(C) Psychonomic Society, Inc. 2011

\begin{abstract}
We propose a two-stage method for comparing standardized coefficients in structural equation modeling (SEM). At stage 1, we transform the original model of interest into the standardized model by model reparameterization, so that the model parameters appearing in the standardized model are equivalent to the standardized parameters of the original model. At stage 2, we impose appropriate linear equality constraints on the standardized model and use a likelihood ratio test to make statistical inferences about the equality of standardized coefficients. Unlike other existing methods for comparing standardized coefficients, the proposed method does not require specific modeling features (e.g., specification of nonlinear constraints), which are available only in certain SEM software programs. Moreover, this method allows researchers to compare two or more standardized coefficients simultaneously in a standard and convenient way. Three real examples are given to illustrate the proposed method, using EQS, a popular SEM software program. Results show that the proposed method performs satisfactorily for testing the equality of standardized coefficients.
\end{abstract}

We would like to thank Prof. G. Francis, the action editor of Behavior Research Methods, and two anonymous reviewers for their comments and suggestions.

Electronic supplementary material The online version of this article (doi:10.3758/s13428-011-0088-6) contains supplementary material, which is available to authorized users.

J. L. Y. Kwan · W. Chan $(\bowtie)$

Department of Psychology,

The Chinese University of Hong Kong,

Shatin, New Territories, Hong Kong

e-mail: wchan@psy.cuhk.edu.hk
Keywords Standardization - Model reparameterization . Structural equation modeling

In social science research, scales of measurement are usually arbitrary, and using the same metric for all variables is rare. Social researchers often have to consider the issue of standardization. Generally speaking, unstandardized coefficients are model parameter estimates based on the analysis of raw data. In contrast, standardized coefficients are model parameter estimates based on the analysis of standardized data, in the sense that all variables are supposed to have unit variance. Standardized data are affected less by the scales of measurement and can be used to compare the relative impact of variables that are incommensurable (i.e., measured in different units on the same/different scales). In multiple regression analysis, for example, researchers are advised to use the beta weights (i.e., standardized regression coefficients) for comparing the relative importance of different incommensurable independent variables for the outcome (Fox, 1997). In fact, there are two different contexts for comparing standardized coefficients. The first is a within-group comparison in which standardized coefficients across different variables are compared within a single sample. The second is a between-group comparison in which standardized coefficients for the same variables are compared across different samples. This article deals primarily with the first context, because it is more likely for different variables to be incommensurable within a single sample and the standardization issue is of particular relevance to this context.

To put the standardization issue into perspective, let us consider an example by using data from the Organization for Economic Cooperation and Development (OECD) Programme for International Student Assessment (PISA) 
2006 (OECD, 2009). ${ }^{1}$ Specifically, a regression model is proposed for examining the effects of parental educational level $(V 2)$ and child's home possession $(V 3)$ on a child's educational resources at home (V4). Table 1 summarizes the covariance matrix of the variables on a sample of 200 Hong Kong students.

To evaluate the relative importance of the two predictors for the dependent variable, we test the equality of the two regression coefficients by using a likelihood ratio (LR) test.A standard path model analysis is conducted to test the unstandardized hypothesis $\mathrm{H}_{0}: \gamma_{1}=\gamma_{2}$, whereas the proposed method, which will be explained in the later section, is applied to the test of the standardized hypothesis $\mathrm{H}_{0}: \gamma_{1}$ * = $\gamma_{2}{ }^{*}$. The analysis is done by using EQS 6.1. ${ }^{2}$ Table 2 (lower panel) shows the LR test results. In the unstandardized condition $\left(\mathrm{H}_{0}: \gamma_{1}=\gamma_{2}\right)$, the result is significant, with $\Delta \chi^{2}=$ 6.002, $\Delta d f=1, p<.05$, suggesting that the effects of $V 2$ and $V 3$ on $V 4$ are different from each other. However, in the standardized condition $\left(\mathrm{H}_{0}: \gamma_{1}{ }^{*}=\gamma_{2}{ }^{*}\right)$, the result is not significant, with $\Delta \chi^{2}(1)=0.011, \Delta d f=1, p>.05$, suggesting that the standardized effects of $V 2$ and $V 3$ on $V 4$ are the same. The seemingly inconsistent findings indicate the fact that these are, indeed, two different tests assessing the equality of different model parameters. If $\mathrm{H}_{0}: \gamma_{1}=\gamma_{2}$ is the null hypothesis we test in the unstandardized condition, the null hypothesis in the standardized condition will become $\mathrm{H}_{0}: \gamma_{1}{ }^{*}=\gamma_{2}{ }^{*}$, where $\gamma_{1}^{*}=\frac{S D(V 2)}{S D(V 4)} \gamma_{1}$ and $\gamma_{2}{ }^{*}=\frac{S D(V 3)}{S D(V 4)} \gamma_{2}$. It is clear that if the standard deviations (SDs) of $V 2$ and $V 3$ are similar (i.e., $V 2$ and $V 3$ are measured by comparable metrics), the two tests will lead to similar results. However, $V 2$ and $V 3$ are measured in very different metrics in this example. The variance of $V 2(=9.07)$ is about ten times larger than the variance of $V 3(=0.84)$, and, therefore, the two tests lead to a very different statistical conclusion.

Behavioral researchers are often interested in comparing the effects of different variables. In some situations, one can draw meaningful conclusions only by comparing different variables in the standardized metric and the associated standardized coefficients thereof. As in the example above, the impact of parental educational level and child's home possession are, in fact, similar if we consider the two effects in a standardized metric. Unfortunately, we sometimes may

\footnotetext{
${ }^{1}$ The Programme for International Student Assessment (PISA) is a triennial international assessment of 15-year-old school children's capabilities in reading literacy, mathematics literacy, and science literacy. The database can be accessed through the PISA Webpage (www.pisa.oecd.org). In this article, data from PISA collected in 2006 was used. PISA 2006 was administrated in 57 countries/economies (OECD 2009). Two hundred cases from Hong Kong were randomly selected from the PISA 2006 data, and five variables are used in this article.

${ }^{2}$ EQS program codes for fitting the models are available as an Electronic Supplementary Material.
}

fail to recognize the fact that the tests for $\mathrm{H}_{0}: \gamma_{1}=\gamma_{2}$ and $\mathrm{H}_{0}: \gamma_{1}^{*}=\gamma_{2} *$ are different and, therefore, may attempt to make statistical conclusions about the standardized coefficients on the basis of the comparison of their unstandardized counterparts. This could be problematic because, as was shown in our previous example, the two tests could lead to a very different result.

Structural equation modeling (SEM) is becoming an increasingly important statistical technique among applied researchers because of its flexibility for studying a variety of different models (e.g., Hershberger, 2003; Tremblay \& Gardner, 1996). Moreover, the development of userfriendly and powerful software programs has contributed significantly to the popularity of this technique (Guo, Perron, \& Gillespie, 2009). Nevertheless, different SEM programs are equipped with different programming features, which may be critical for addressing a particular research question, such as the comparison of standardized coefficients. The aim of this study, therefore, is to propose a general method for comparing the standardized coefficients in SEM based on the idea of model reparameterization.

Although there currently exist other methods for comparing standardized coefficients in SEM, we argue that the proposed method is a more general and flexible one. These existing methods are known to be program specific, in the sense that their implementation depends critically on the special features, such as the specification of nonlinear constraints and the availability of an overall test for comparing three or more parameters simultaneously, which some popular programs are still lacking. Our proposed method, on the other hand, does not require any advanced programming features except the basic functions, and it is, therefore, compatible with all major SEM software programs. We believe that one issue that prevents applied researchers from comparing standardized coefficients is that the researchers are limited by the inability of their SEM software programs to provide the relevant tests. In the next section, we will briefly summarize the existing methods for comparing standardized coefficients in SEM. The proposed method is then given in the third section. Three real examples that illustrate the proposed method will be considered in the fourth section. A discussion and conclusions will be provided in the final section.

\section{Comparing standardized coefficients in structural equation modeling}

Built-in functions by different software programs

The early development of SEM software programs primarily focused on parameter estimation and statistical inference of unstandardized parameters. Although many programs nowa- 
Table 1 Sample covariance (below diagonal) /correlation (above diagonal) matrix for Hong Kong students from PISA2006 (OECD, 2009) $(N=200)$

\begin{tabular}{|c|c|c|c|c|c|}
\hline & $\begin{array}{l}\text { Parental occupational } \\
\text { status }(V 1)\end{array}$ & $\begin{array}{l}\text { Parental education } \\
\text { level }(V 2)\end{array}$ & $\begin{array}{l}\text { Home possession } \\
(V 3)\end{array}$ & $\begin{array}{l}\text { Home educational } \\
\text { resources }(V 4)\end{array}$ & $\begin{array}{l}\text { Reading } \\
\text { scores }(V 5)\end{array}$ \\
\hline \multirow[t]{2}{*}{$V 1$} & 1.0000 & 0.6606 & 0.3547 & 0.1570 & 0.2310 \\
\hline & 226.2577 & & & & \\
\hline \multirow[t]{2}{*}{$V 2$} & & 1.0000 & 0.3743 & 0.3962 & 0.2110 \\
\hline & 29.9232 & 9.0692 & & & \\
\hline \multirow[t]{2}{*}{$V 3$} & & & 1.0000 & 0.3887 & 0.2806 \\
\hline & 4.8812 & 1.0312 & 0.8371 & & \\
\hline \multirow[t]{2}{*}{$V 4$} & & & & 1.0000 & 0.2391 \\
\hline & 1.9878 & 1.0043 & 0.2993 & 0.7084 & \\
\hline \multirow[t]{3}{*}{$V 5$} & & & & & 1.0000 \\
\hline & 271.1429 & 49.5848 & 20.0337 & 15.7012 & \\
\hline & & & & & 6088.8281 \\
\hline
\end{tabular}

days have built-in functions for handling standardized parameters, these functions are still very limited. For example, the current versions of AMOS (Arbuckle, 2007), EQS (Bentler, 1995), and LISREL (Jöreskog \& Sörbom, 1996) can provide only the standardized parameter estimates, without their standard errors (SEs). Mplus 5.0 or above (Muthén \& Muthén, 2007) provides both the standardized parameter estimates and their SEs. The program, however, does not report the covariances among the standardized parameter estimates, which are also important for the test because the standardized coefficients being compared are generally not independent. Moreover, none of these pro- grams has any built-in function that allows their users to compare the standardized coefficients directly.

Phantom variables approach

Cheung (2009b) has given a detailed description of how to construct the confidence intervals on the difference between two standardized coefficients with the use of phantom variables. A phantom variable is a latent variable without observed indicators and has no residual (Rindskopf, 1984). It can be used to trick model-fitting programs into imposing constraints that are not normally within their repertoire

Table 2 Example of the comparison of unstandardized results and standardized results

\begin{tabular}{|c|c|c|c|c|c|c|c|c|}
\hline \multirow[b]{3}{*}{ Parameter } & \multicolumn{4}{|c|}{ Unstandardized estimates ${ }^{\mathrm{a}}$} & \multicolumn{4}{|c|}{ Standardized estimates ${ }^{\mathrm{b}}$} \\
\hline & \multicolumn{2}{|c|}{ Unconstrained model } & \multicolumn{2}{|c|}{ Constrained model ${ }^{\mathrm{c}}$} & \multicolumn{2}{|c|}{ Unconstrained model } & \multicolumn{2}{|c|}{ Constrained model ${ }^{\mathrm{d}}$} \\
\hline & Est. & $S E$ & Est. & $S E$ & Est. & $S E$ & Est. & $S E$ \\
\hline$V 2 \rightarrow V 4\left(\gamma_{1} / \gamma_{1}^{*}\right)$ & 0.081 & 0.019 & 0.109 & 0.015 & 0.292 & 0.065 & 0.286 & 0.032 \\
\hline$V 3 \rightarrow V 4\left(\gamma_{2} / \gamma_{2} *\right)$ & 0.257 & 0.062 & 0.109 & 0.015 & 0.280 & 0.065 & 0.286 & 0.032 \\
\hline$V 3 \leftrightarrow V 4\left(\phi / \phi^{*}\right)$ & 1.031 & 0.209 & 1.031 & 0.209 & 0.374 & 0.061 & 0.374 & 0.061 \\
\hline Model chi-square $\left(\chi^{2}\right)$ & \multicolumn{2}{|l|}{0.000} & \multicolumn{2}{|l|}{6.002} & \multicolumn{2}{|l|}{0.000} & \multicolumn{2}{|l|}{0.011} \\
\hline \multirow[t]{2}{*}{ Degree of freedom $(d f)$} & 0 & & 1 & & 0 & & 1 & \\
\hline & \multicolumn{4}{|c|}{ Testing $\mathrm{H}_{0}: \gamma_{1}=\gamma_{2}$} & \multicolumn{4}{|c|}{ Testing $\mathrm{H}_{0}: \gamma_{1} *=\gamma_{2} *$} \\
\hline Likelihood ratio test & \multicolumn{4}{|c|}{$\Delta \chi^{2}=6.002, \Delta d f=1, p=.014$} & \multicolumn{4}{|c|}{$\Delta \chi^{2}=0.011, \Delta d f=1, p=.916$} \\
\hline \multirow[t]{3}{*}{ Wald test ${ }^{\mathrm{e}}$} & \multicolumn{4}{|c|}{$\gamma_{2}-\gamma_{1}=0.176$} & \multicolumn{4}{|c|}{$\gamma_{2}^{*-} \gamma_{1} *=-0.012$} \\
\hline & \multicolumn{4}{|c|}{$S E=0.071$} & \multicolumn{4}{|c|}{$S E=0.113$} \\
\hline & \multicolumn{4}{|c|}{$\left(\gamma_{2}-\gamma_{1}\right) / S E=2.475, p=.013$} & \multicolumn{4}{|c|}{$\left(\gamma_{2}^{*}-\gamma_{1} *\right) / S E=-0.107, p=.915$} \\
\hline
\end{tabular}

Est. = parameter estimate; $S E=$ standard error

${ }^{a}$ Original model of interest was fitted to the observed data by using EQS6.1

${ }^{\mathrm{b}}$ We first transformed the original model into the standardized model by using the proposed method and fitted the model to the observed data by using EQS 6.1

${ }^{\mathrm{c}}$ Constrained model is fitted under $\mathrm{H}_{0}: \gamma_{1}=\gamma_{2}$

${ }^{\mathrm{d}}$ Constrained model is fitted under $\mathrm{H}_{0}: \gamma_{1} *=\gamma_{2} *$

${ }^{\mathrm{e}}$ Wald test resultsare based on the analysis by using Mplus5.2 
(Loehlin, 2004). Many SEM programs have functions that help to simplify the model specification involving phantom variables. For example, LISREL has an AP function (Jöreskog \& Sörbom, 1996), and Mplus has a MODEL CONSTRAINT option (Muthén \& Muthén, 2007) for creating additional parameters. By defining an additional parameter as the difference between two standardized coefficients, we can readily obtain the parameter estimate and its $S E$ and can use the Wald statistic to test its statistical significance (cf. Cheung, 2009b).

Although the phantom variable approach provides a general solution for comparing standardized coefficients in SEM, it can be implemented only by using a specific class of SEM software programs. First, a nonlinear constraint is an essential feature of the phantom variables approach, because the difference in two standardized coefficients (i.e., the additional parameter) is defined as a nonlinear function of the basic model parameters. As a result, users must have an SEM program that supports model fitting with nonlinear constraints (e.g., LISREL and Mplus) in order to implement the method. Unfortunately, not many applied researchers can get access to these programs freely. In fact, most local academic departments and research institutes can afford to support and maintain only one SEM program due to various practical reasons, such as limited resources, personal preference, and faculty training. It is not feasible for them to switch from one SEM program to another. Furthermore, many reported SEM studies indicated that their analyses were based primarily on AMOS or EQS, which currently do not support the specification of nonlinear constraints. For example, a review by Guo et al. (2009) showed that around $50 \%$ of the studies used either AMOS or EQS but only around 3\% of those used Mplus. Similarly, another review by Jackson, Gillaspy, and Purc-Stephenson (2009) suggested that the figures were around $40 \%$ and $7 \%$, respectively.

Second, the phantom variable approach basically defines an additional parameter as the difference of two standardized coefficients and tests its value against zero by using the Wald statistic. Consequently, whether this approach can be extended to the comparison of three or more standardized coefficients simultaneously will depend further on the availability of an overall test for all the additional parameters concerned. As far as we know, most of the SEM programs fail to perform an overall test like this, except Mplus. This further narrows down one's choices of programs for comparing standardized coefficients.

\section{The proposed method}

The proposed method uses model reparameterization for standardizing model parameters. The idea of model reparameterization is to transform the hypothesized model into a set of successive covariance-equivalent models that share the same implied covariance matrix as the original model. As a result, a coefficient that does not exist as a model parameter in the original model becomes a model parameter in the final transformed model. Chan's (2007) sequential model-fitting method for comparing the indirect (mediation) effects in SEM demonstrated one of the usages of the model reparameterization technique. Our proposed method applies the model reparameterization technique to the standardization of model parameters and demonstrates another usage of the technique.

The proposed method adopts a two-stage approach for comparing standardized coefficients. At stage 1, we first transform the original model (M1) into the standardized model (M2) by reparameterization so that the path coefficients as described in the transformed model are equivalent to the standardized path coefficients of the original model. Once the standardized coefficients appear as free model parameters in M2, we can test their differences. Hence, at stage 2, we compare the standardized coefficients by imposing appropriate equality constraint(s) on the parameters of interest in M2 and perform statistical inference on the basis of the LR test. In the following section, we give a detailed description of how to transform the original model into the standardized model at stage 1 .

\section{General framework of model transformation at stage 1}

Like other SEM analyses, we first define a given model that is of theoretical interest. We label this model as the original model, M1. Figure 1a show M1 with $k$ effects acting on $Y$, where $\gamma_{1}, \gamma_{2}, \ldots \gamma_{k}$ are the unstandardized path coefficients, $\phi_{i j}$ is the covariance between $X i$ and $X j$, and $E$ is the error term. Without loss of generality, all variables are assumed to have zero means. The model equation of the original model in standardized form can be written as follows:

$$
\begin{aligned}
Y & =\sum_{i}^{k} \gamma_{i} X i+E \\
\frac{Y}{S D(Y)} & =\sum_{i}^{k}\left(\frac{S D(X i) \gamma_{i}}{S D(Y)} \times \frac{X i}{S D(X i)}\right)+\frac{E}{S D(Y)}
\end{aligned}
$$

Our task is, therefore, to transform the original model so that the standardized coefficients, $\gamma_{i}{ }^{*}=\frac{S D(X)}{S D(Y)} \gamma_{i}$, become model parameters of the standardized model, M2.

Figure $1 \mathrm{~b}$ shows a half-transformed model of M1. We first transform the model by regressing the $(k+1)$ observed variables on $(k+1)$ dummy latent variables (DLVs), $F 1$, $F 2, \ldots, F k$, and $F Y$, which are manipulated to have unit variance. $D$ is the disturbance term. Chan (2007) has used the term $D L V$ to denote the variable that was used to factorize the original mediator in the sequential modelfitting method. The function of DLV is similar to 
(a)

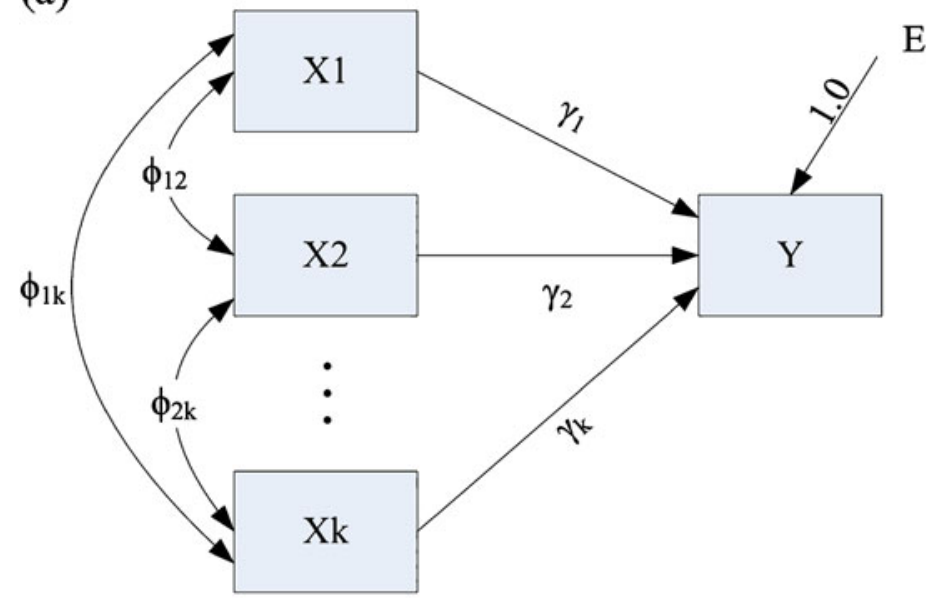

(b)

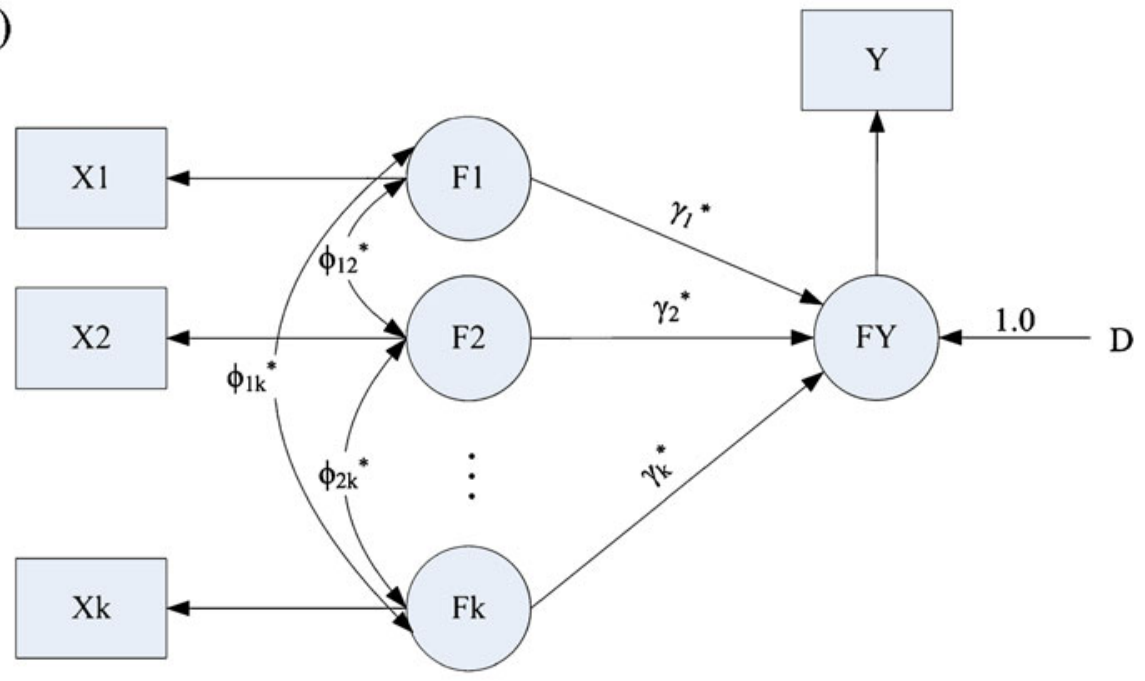

(c)

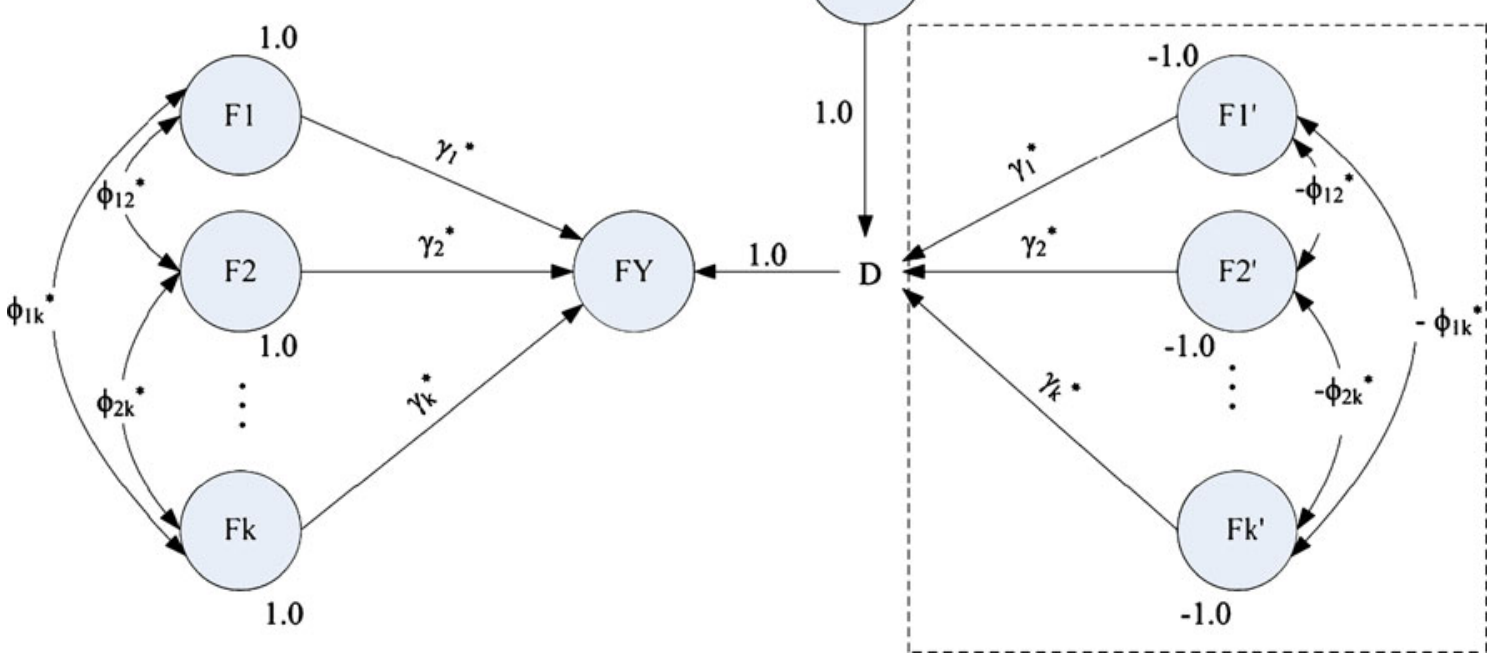

Fig. 1 A general regression model with $k$ effects on $Y$. a Original model (M1). b Half -transformed model. c Final standardized model (M2). Observed variables $(X 1, \ldots, X k$ and $Y)$ are omitted in panel $\mathrm{c}$ 
Rindskopf's (1984) concept of a phantom variable. ${ }^{3}$ The model equation of the half-transformed model is as follows:

$F Y=\sum_{i}^{k} \gamma_{i}^{*} F_{i}+D$

By standardizing $F 1, F 2, \ldots, F k$, and $F Y$ (i.e., all the variables have unit variance), Eq. 2 will be equivalent to Eq. 1. In other words, $\gamma_{i}^{*}$ will be equal to the standardized path coefficients of the original model. Since $F 1$ to $F k$ are independent variables, we can fix their variances directly as 1.0 in SEM. However, $F Y$ is a dependent variable, and its variance does not exist as a free parameter in SEM, so we cannot fix its variance directly. Therefore, the question becomes how we can standardize FY. From Eq. 2, the variance of $F Y$ is

$$
\begin{aligned}
\operatorname{var}(F Y) & =\operatorname{var}\left(\sum_{i}^{k} \gamma_{i}^{*} F i+D\right) \\
& =\sum_{i}^{k} \gamma_{i}^{* 2}+2 \sum \sum_{i \neq j} \gamma_{i}^{*} \gamma_{j}^{*} \phi_{i j}^{*}+\operatorname{var}(D) \\
& =g(\theta)+\operatorname{var}(D)
\end{aligned}
$$

where $\phi_{i j}{ }^{*}$ is the covariance between $F i$ and $F j, \theta$ is a vector of unknown model parameters, $g(\theta)=\sum_{i}^{k} \gamma_{i}^{* 2}+$ $2 \sum \sum_{i \neq j} \gamma_{i}^{*} \gamma_{j}{ }^{*} \phi_{i j}{ }^{*}$ is defined as the total variances and covariances due to the antecedent variables, and $\operatorname{var}(D)$ is the disturbance variance. If $\operatorname{var}(D)=1-g(\theta)$, then $\operatorname{var}(F Y)$ will become 1.0 as $\operatorname{var}(F Y)=g(\theta)+[1-g(\theta)]=1.0$.

In programs such as LISREL and Mplus, we can fix the disturbance variance by using nonlinear constraints, but our aim is to propose a method that does not involve nonlinear constraints. Therefore, we need to further transform the model into the final standardized model (M2) by regressing the disturbance term on a phantom variable, $F 999,{ }^{4}$ with unit variance and $k$ image latent variables $\left(F 1^{\prime}, F 2^{\prime} \ldots F k^{\prime}\right)$, with variance $=-1.0$. We labeled $F 1^{\prime}, F 2^{\prime} \ldots F k^{\prime}$ as image latent variables and the structure formed by them as the image structure of the effects on FY. An image structure is

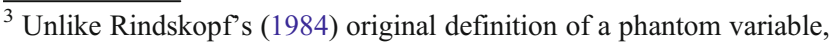
which is a latent variable with no observed indicators, a DLV has other variables loaded on it. The concept of DLV, rather than phantom variable, is adopted here because the latent variables in the standardized model have the original observed variables loaded on them.

${ }^{4}$ Although the general function of phantom variables in both the phantom variable approach and the proposed method is to impose parameter constraints on the model, they serve different roles in the two methods. The phantom variable is used for creating a new parameter in the phantom variable approach, whereas the phantom variable $F 999$ is used for reparameterizing the disturbance variance here.
}

defined by the following four properties: (1) The image structure has the same structural form as the target structure; (2) the path coefficients of the image structure are the same as the corresponding path coefficients of the target structure; (3) $\operatorname{var}\left(F i^{\prime}\right)=-\operatorname{var}(F i)$; and (4) $\operatorname{cov}\left(F i^{\prime}, F j^{\prime}\right)=-\operatorname{cov}(F i, F j)$. As can be seen in Fig. 1c, $F 1^{\prime}$ to $F k^{\prime}$ has the same structure as $F 1$ to $F k$. The path leading from $F i^{\prime}$ to $D$ is the same as the path leading from $F i$ to $F Y$ (i.e., $\left.\gamma_{i}^{*}\right)$. $\operatorname{Var}\left(F i^{\prime}\right)=-1$, which is the image of $\operatorname{var}(F i)=1$; and $\operatorname{cov}\left(F i^{\prime}, F j^{\prime}\right)=-\phi_{i j}{ }^{*}$, which is the image of $\operatorname{cov}(F i, F j)=\phi_{i j}$. In theory, the variance of a random variable could not be negative. In this case, however, we pragmatically consider negative unit variance of $F i^{\prime}$ to generate the desired variance of $D$.

In Fig. 1c, the effect of $F i^{\prime}$ on $D\left(\gamma_{i}^{*}\right)$ is equal to the effect of $F i$ on $F Y$, and the path leading from $F 999$ to $D$ is always fixed at 1.0. By fixing $\operatorname{var}(F 999)=1.0$, $\operatorname{var}\left(F i^{\prime}\right)=-1.0$, and $\operatorname{cov}\left(F i^{\prime}, F j^{\prime}\right)=-\phi_{i j}{ }^{*}$, we will have

$$
\begin{aligned}
D & =F 999+\sum_{i}^{k} \gamma_{i}^{*} F_{i}^{\prime} \\
\operatorname{var}(D) & =\operatorname{var}(F 999)+\sum_{i}^{k} \gamma_{i}^{* 2} \operatorname{var}\left(F i^{\prime}\right)+2 \sum \sum_{i \neq j} \gamma_{i}^{*} \gamma_{j}^{*}\left(-\phi_{i j}{ }^{*}\right) \\
& =1.0-\left(\sum_{i}^{k} \gamma_{i}^{* 2}+2 \sum \sum_{i \neq j} \gamma_{i}^{*} \gamma_{j}^{*} \phi_{i j}^{*}\right)=1.0-g(\theta)
\end{aligned}
$$

In short, two sources of effects act on the disturbance term, $D$, in M2: (1) the effect of phantom variable, $F 999$, with unit variance, and (2) the effects of the image structure $\left(F 1^{\prime}\right.$ to $\left.F k^{\prime}\right)$, which make up a total variance of $-g(\theta)$.By substituting Eq. 4 into Eq. 3, we can see that the variance of the dependent latent variable, $F Y$, is fixed at 1.0 nonstochastically:

$\operatorname{var}(F Y)=g(\theta)+\operatorname{var}(D)=g(\theta)+1-g(\theta)=1.0$.

To summarize, two important criteria need to be observed when a model transformation is performed. First, the variances of the DLVs in M2 must be fixed at 1.0 nonstochastically. Second, M2 must have the same implied covariance structure as the original model. Once we successfully transform the original model into the standardized model at stage 1, comparing the standardized coefficients using the LR test at stage 2 is straightforward.

\section{Real examples}

Three real examples are considered in order to illustrate the proposed method using EQS (Bentler, 1995). Readers who are interested in working on these examples can also download the complete EQS program 
codes (see Electronic Supplementary Material). The first two examples use a sample that consists of 200 (Hong Kong) cases randomly selected from the PISA 2006 data set (OECDS, 2009) on five variables. They are, namely, parental occupational status $(V 1)$, parental educational level $(V 2)$, child's home possession (V3), child's home educational resources (V4), and child's reading ability (V5). Table 1 summarizes the sample covariance matrix of the variables.

Example 1: A regression model with three predictors

Stage 1 Figure 2a shows the original model, M1. In this example, a regression model with three predictors is considered. Specifically, we attempt to compare the standardized effects of parental occupational status $\left(\gamma_{1} *\right)$, parental educational level $\left(\gamma_{2}{ }^{*}\right)$, and child's home possession $\left(\gamma_{3}^{*}\right)$ on child's reading ability (V5). The model is fitted to the observed data by using EQS6.1 for Windows. Since M1 is a saturated model with 0 degrees of freedom, it has a perfect fit with model chi-square, $\chi^{2}=0$.

We follow the general framework to transform M1 into the standardized model, M2. Figure $2 b$ depicts the standardized model, M2. First, each observed variable is regressed on a DLV ( $F 1$ to $F 4$ ). Variances of $F 1$ to $F 3$ are fixed at 1.0. $F 5$ is the disturbance term of $F 4^{5}$. F999 is the phantom variable with unit variance, and the path from $F 999$ to $F 5$ is fixed at 1.0. $F 6=F 1^{\prime}, F 7=F 2^{\prime}$, and $F 8=F 3^{\prime}$ are image latent variables with negative unit variance, and they form the image structure of $F 1, F 2$, and $F 3$.

Second, M2 is fitted to the observed data. ${ }^{6}$ The path coefficients of the image latent variables $F 6, F 7$, and $F 8$ are constrained to be equal to the coefficients of the corresponding target variables $F 1, F 2$, and $F 3$. Covariance between the image latent variables is constrained to be equal to the negative of the covariance between the target variables. Six linear constraints (three on $\gamma_{i}^{*}$ 's and three on $\phi_{i j}{ }^{*}$ 's) are imposed on the model altogether. Again, the model has a perfect fit with model chi-square, $\chi^{2}=0, d f=$ 0 . Table 3 shows the parameter estimates and their standard errors $(S E \mathrm{~s})$ in M2 (under the heading "reparameterization"). By comparing the parameter estimates and their $S E \mathrm{~s}$

\footnotetext{
${ }^{5}$ In EQS, the disturbance term can appear only as an exogenous variable, which cannot be regressed on other variables. To solve this problem, we rename the disturbance term as $F 5$, an ordinary latent factor.

${ }^{6}$ In all examples, the standardized model fails to converge initially with the default starting values in EQS. The problem is solved by providing better starting values to the algorithm. As a general guideline, one can first fit the original model to obtain the standardized parameter estimates and use them as the starting values in the transformed model. AMOS, on the other hand, does not have such a problem with their default starting values.
}

with the standardized parameter estimates and their $S E \mathrm{~s}$ reported by Mplus (with a built-in function) and LISREL (by using the phantom variables approach ${ }^{7}$ ), they are perfectly comparable with each other. ${ }^{8}$ In other words, the original model has been successfully transformed into the standardized model, and the path coefficients that appear in M2 are equivalent to the standardized coefficients of the original model, M1.

Stage 2 To test the equality of the three standardized coefficients from $V 1, V 2$, and $V 3$ to $V 5$, we fit a constrained model under $\mathrm{H}_{0}: \gamma_{1}{ }^{*}=\gamma_{2}{ }^{*}=\gamma_{3} *$ by imposing two linear equality constraints: (1) $F 1 \rightarrow F 4=F 3 \rightarrow F 4$ and (2) $F 2 \rightarrow$ $F 4=F 3 \rightarrow F 4$. The model chi-square is $\chi^{2}=2.070, d f=2$, $p=.355$. Table 4 shows the model chi-squares and the parameter estimates of the constrained and unconstrained models. We compare the chi-square of the constrained model with that of the unconstrained model. The LR test gives $\Delta \chi^{2}=2.070-0=2.070, \Delta d f=2-0=2, p>.05$, suggesting that there is no significant difference among the standardized regression coefficients. Hence, we can conclude that the relative effects of parental occupational status, parental educational level, and child's home possession on child's reading ability are the same.

Example 2: A path model with three antecedent variables, one mediator, and one outcome variable

Stage 1 We further hypothesize that the child's educational resources at home (V4) mediates the relationships of parental occupational status $(V 1)$, parental educational level $(V 2)$, and child's possession at home $(V 3)$ with child's reading ability (V5). Therefore, we define the original model, M1 (Fig. 3a), as a five-variable path model with $V 1, V 2$, and $V 3$ as the antecedent variables, $V 4$ as the mediator, and $V 5$ as the outcome variable. In this example, the aim is to compare the standardized effects of $V 1, V 2$, and $V 3$ on $V 4$. When $\gamma_{1}{ }^{*}=$ $\gamma_{2}{ }^{*}=\gamma_{3}{ }^{*}$, the indirect effects on $V 5$ are said to be equal. We fit M1 to the observed data using EQS. The chi-square goodness-of-fit statistic is $\chi^{2}=13.218, d f=3, p<.01$.

\footnotetext{
$\overline{{ }^{7} \text { By following }}$ the phantom variable approach, we create additional parameters to define the standardized parameters (using $A P$ keyword) in LISREL. The estimated SEs of the additional parameters, which are equivalent to the estimated $S E$ s of the corresponding standardized parameters, are reported.

${ }^{8}$ In addition, we fit the same original model (M1) to the correlation matrix by using the SEPATH module (Steiger, 1995) in Statistica 9.0, which has a built-in option for implementing a constrained estimation method (Browne, 1982; Browne \& Mels, 1992; Mels, 1989), and the program returns the same standardized results (not presented here), as compared with those of our proposed method (including estimates, $S E$ s, test statistics, model fits, and chi-square change).
} 
(a)

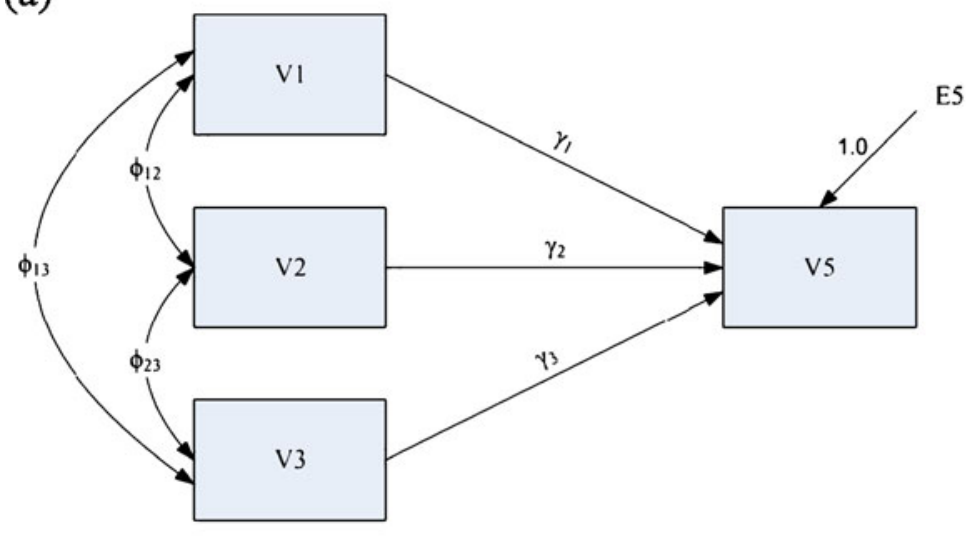

(b)

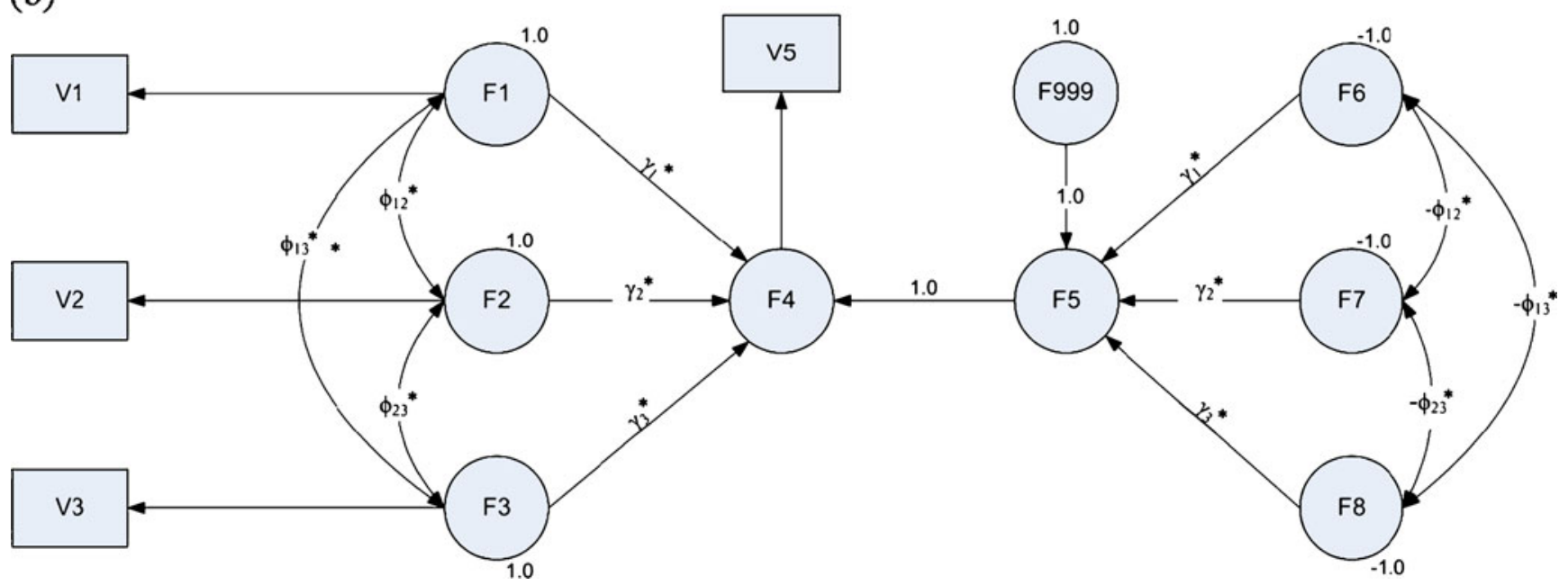

Fig. 2 Models in Example 1. a Original model (M1). b Standardized model (M2). $V 1=$ parental occupational status, $V 2=$ parental educational level, $V 3=$ child's home possession, $V 5=$ reading scores. Labels for parameters of interest are printed

Table 3 Summary of the standardized parameter estimates and their estimated standard errors in Example 1 by analysis using different structural equation modeling programs and approaches

\begin{tabular}{|c|c|c|c|c|c|c|c|c|c|c|}
\hline \multirow{3}{*}{$\begin{array}{l}\text { Program } \\
\text { Method } \\
\text { Parameter }\end{array}$} & \multicolumn{6}{|c|}{ EQS6.1 } & \multirow{2}{*}{\multicolumn{2}{|c|}{$\frac{\text { Mplus5.2 }}{\text { Built-in }^{\mathrm{b}}}$}} & \multirow{2}{*}{\multicolumn{2}{|c|}{$\begin{array}{l}\text { LISREL8.8 } \\
\text { Phantom variable }\end{array}$}} \\
\hline & \multicolumn{2}{|c|}{ Reparameterization } & \multicolumn{2}{|c|}{ Correlation } & \multicolumn{2}{|c|}{ Bootstrap $^{a}$} & & & & \\
\hline & Est. & $S E$ & Est. & $S E$ & Est. & $S E$ & Est. & $S E$ & Est. & $S E$ \\
\hline$\gamma_{1}^{*}$ & .121 & .090 & .121 & .091 & .125 & .083 & .121 & .090 & .121 & .091 \\
\hline$\gamma_{2}{ }^{*}$ & .049 & .091 & .049 & .091 & .044 & .088 & .049 & .091 & .049 & .092 \\
\hline$\gamma_{3}^{*}$ & .219 & .072 & .219 & .073 & .222 & .074 & .219 & .072 & .219 & .072 \\
\hline$\phi_{12} *$ & .661 & .040 & .661 & .085 & .660 & .037 & .661 & .040 & .661 & .040 \\
\hline$\phi_{13}{ }^{*}$ & .355 & .062 & .355 & .075 & .353 & .057 & .355 & .062 & .355 & .062 \\
\hline$\phi_{23}{ }^{*}$ & .374 & .061 & .374 & .076 & .377 & .057 & .374 & .061 & .374 & .061 \\
\hline
\end{tabular}

Est. = parameter estimate; $S E=$ standard error; Standard errors that deviate substantially from estimates of other methods are in bold face.

${ }^{\mathrm{a}}$ Regular (i.e., completely nonparametric) bootstrap is used; replication $=1,000$

${ }^{\mathrm{b}}$ The StdYX standardization are reported here. StdYX uses the variances of the continuous latent variables, as well as the variances of the background and outcome variables for standardization (L. K. Muthèn \& B. O. Muthèn, 2007) 
Table 4 Hypothesis testing results based on the unstandardized model (M1) and standardized model (M2) in Example 1

\begin{tabular}{|c|c|c|c|c|c|c|c|c|}
\hline \multirow{3}{*}{$\begin{array}{l}\text { Model } \\
\text { Parameter }\end{array}$} & \multicolumn{4}{|c|}{ M1(Unstandardized) } & \multicolumn{4}{|c|}{ M2 (Standardized) } \\
\hline & \multicolumn{2}{|c|}{ Unconstrained } & \multicolumn{2}{|c|}{ Constrained $^{\mathrm{a}}$} & \multicolumn{2}{|c|}{ Unconstrained } & \multicolumn{2}{|c|}{ Constrained $^{\mathrm{b}}$} \\
\hline & Est. & $S E$ & Est. & $S E$ & Est. & $S E$ & Est. & $S E$ \\
\hline$\gamma_{1} / \gamma_{1}^{*}$ & 0.626 & 0.470 & 1.107 & 0.305 & 0.121 & 0.090 & 0.125 & 0.026 \\
\hline$\gamma_{2} / \gamma_{2} *$ & 01.273 & 2.369 & 1.107 & 0.305 & 0.049 & 0.091 & 0.125 & 0.026 \\
\hline$\gamma_{3} / \gamma_{3} *$ & 18.712 & 6.261 & 1.107 & 0.305 & 0.219 & 0.072 & 0.125 & 0.026 \\
\hline Model chi-square $\left(\chi^{2}\right)$ & \multicolumn{2}{|l|}{0.000} & \multicolumn{2}{|l|}{8.201} & \multicolumn{2}{|l|}{0.000} & \multicolumn{2}{|l|}{2.070} \\
\hline \multirow[t]{2}{*}{ Degree of freedom $(d f)$} & 0 & & 2 & & 0 & & 2 & \\
\hline & \multicolumn{8}{|c|}{ Likelihood ratio test results } \\
\hline Hypothesis & $\Delta \chi^{2}$ & $\Delta d f$ & & $p$ & $\Delta \chi^{2}$ & $\Delta d f$ & & $p$ \\
\hline $\begin{array}{l}\mathrm{H}_{0}: \gamma_{1}=\gamma_{2}=\gamma_{3} / \\
\mathrm{H}_{0}: \gamma_{1}^{*}=\gamma_{2} *=\gamma_{3} *\end{array}$ & $8.201^{*}$ & 2 & & .017 & 2.070 & 2 & & 0.355 \\
\hline
\end{tabular}

Est. = parameter estimate; $S E=$ standard error

*Result is significant at $5 \%$ significance level

${ }^{\text {a }}$ Constrained model is fitted under $\mathrm{H}_{0}: \gamma_{1}=\gamma_{2}=\gamma_{3}$.

${ }^{\mathrm{b}}$ Constrained model is fitted under $\mathrm{H}_{0}: \gamma_{1} *=\gamma_{2}{ }^{*}=\gamma_{3} *$

Following the proposed method, we first transform the model into the standardized model. Although the original model is more complex in this example, the logic of model transformation remains the same. Figure $3 b$ depicts the standardized model. Again, each observed variable is regressed on a DLV ( $F 1$ to $F 5$ ). $F 1$ to $F 3$ are independent variables, and their variances are fixed at 1.0. $F 4$ and $F 5$ are the dependent variables, so we use the disturbance terms, $F 6$ and $F 7$, to standardized $F 4$ and $F 5$, respectively.

For $F 4$, let $g_{4}(\theta)$ be the total variances and covariances due to $F 1$ to $F 3$. Our task, therefore, is to cancel out $g_{4}(\theta)$ by making use of $F 6$ so that $F 4$ can have unit variance. We follow the general framework and regress $F 6$ on $F 888, F 8$, $F 9$, and $F 10$. Hence, $F 888=F 999$ is the phantom variable with unit variance. $F 8=F 1^{\prime}, F 9=F 2^{\prime}$, and $F 10=F 3^{\prime}$ are image latent variables with negative unit variance, and they form the image structure of $F 1, F 2$, and $F 3$ on $F 4$. The path leading from $F 888$ to $F 6$ is fixed at 1.0.

Similarly, for $F 5, g_{5}(\theta)$ is equal to the variance due to $F 4$. To standardized $F 5$, we need to cancel out $g_{5}(\theta)$ by regressing $F 7$ on $F 999$ and $F 11$. F999 is the phantom variable with unit variance, and $F 11=F 4^{\prime}$ is the image latent variable of $F 4$ in this case. The path leading from $F 999$ to $F 7$ is fixed at 1.0. Since the variance of $F 4$ has been fixed at 1.0 indirectly in the previous step, we can cancel the effect of $F 4$ and impose unit variance on $F 5$ by simply fixing the variance of $F 11$ at -1.0 and constraining the path from $F 4$ to $F 5$ to be equal to the path from $F 11$ to $F 7$.

As in Example 1, we fit M2 to the observed data. Four linear equality constraints are imposed on the path coefficients of the image latent variables ( $F 8$ to $F 11)$ and those of their corresponding target variables ( $F 1$ to $F 4$ ). The three covariances among $F 8, F 9$, and $F 10$ are constrained to be equal to the negative of the covariances among $F 1, F 2$, and $F 3$. Seven linear constraints are specified altogether. M2 gives exactly the same chi-square value as $\mathrm{M} 1, \chi^{2}=13.218$, $d f=3, p<.01$, indicating that the two models are covariance equivalent and have the same implied covariance matrix. Table 5 shows the parameter estimates and their $S E$ s, which again are the same as the results given by Mplus and LISREL. ${ }^{9}$

Stage 2 To compare the standardized coefficients of $V 1, V 2$, and $V 3$ on $V 4$, we fit a constrained model by imposing two linear equality constraints: (1) $F 1 \rightarrow F 4=F 3 \rightarrow F 4$ and (2) $F 2 \rightarrow F 4=F 3 \rightarrow F 4$. As is shown in Table 6, the model chisquare is $\chi^{2}=39.097, d f=5$. The LR test gives $\Delta \chi^{2}=$ $39.097-13.218=25.879, \Delta d f=5-3=2, p<.001$, suggesting that the relative effects of parental occupational status, parental education level, and child's home possession on child's educational resources at home are not all equal.Hence, the indirect effects on child's reading ability are also not all equal.

Example 3: A structural model with two antecedent variables and one outcome variable

This example further demonstrates how the proposed method can be applied for comparing standardized coef-

\footnotetext{
${ }^{9}$ Again, the same original model (M1) is fitted to the correlation matrix by using the SEPATH module in Statistica 9.0. The same standardized results (not presented here), as compared with those of our proposed method, are obtained.
} 
(a)

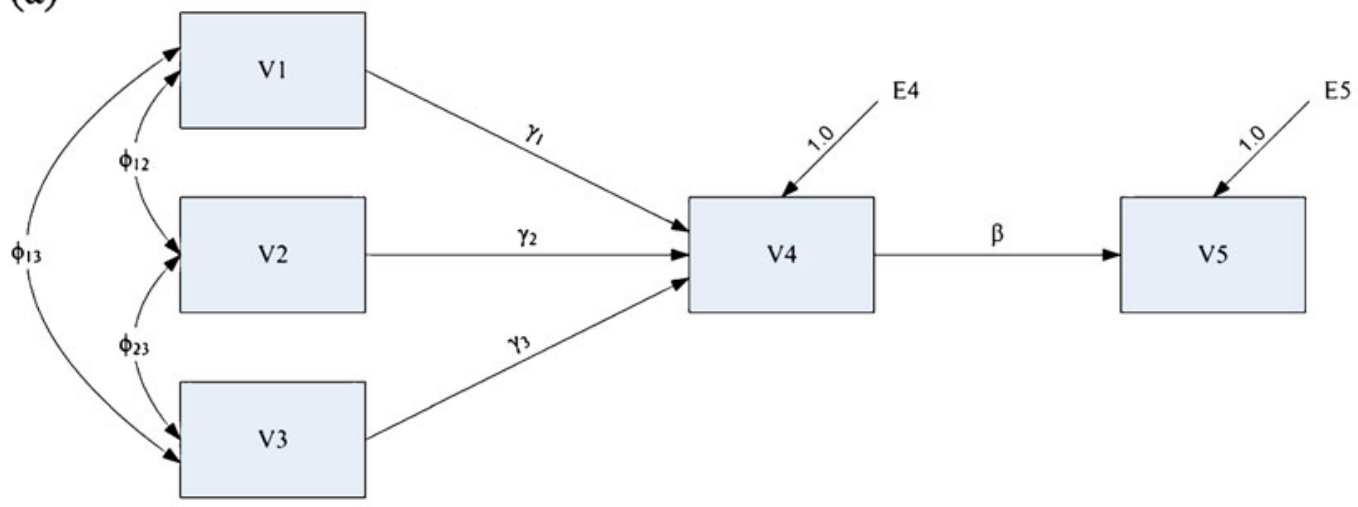

(b)

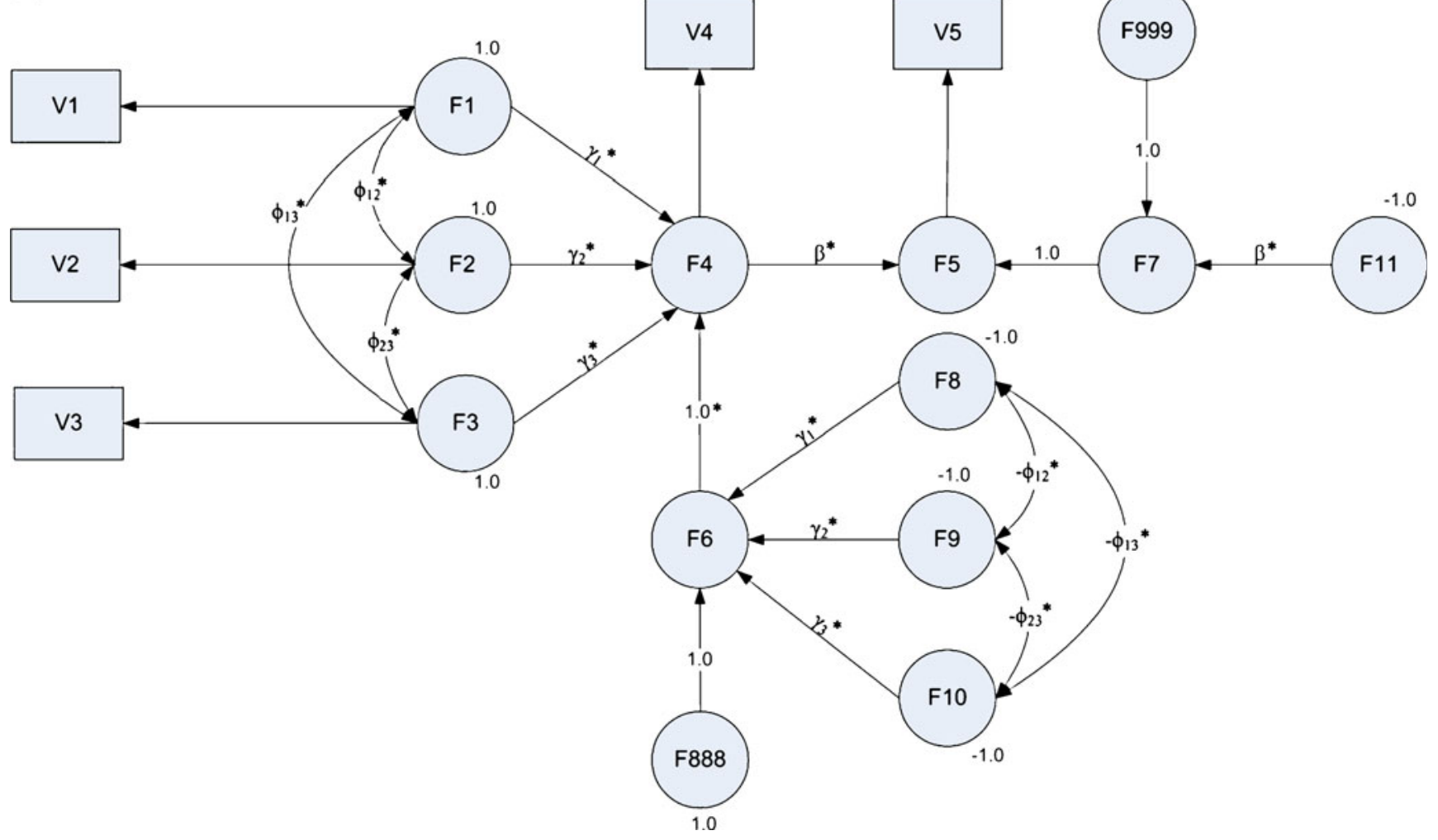

Fig. 3 Models in Example 2. a Original model (M1). b Standardized model (M2). $V 1=$ parental occupational status, $V 2=$ parental educational level, $V 3=$ child's home possession, $V 4=$ child's home educaitonal resources, $V 5=$ reading scores. Labels for parameters of interest are printed

ficients in models with latent variables. Six variables were selected from the dataset in Schoon and Parson (2002), who studied how the social structure influences teenage aspirations and subsequent occupational attainment. They are, namely, examination score ( $V 1)$, highest qualifications ( $V 2)$, job aspiration (V3), educational aspiration (V4), Goldthorpe (V5), and RGSC (V6). The six selected variables together measure three latent factors: educational achievement $(F 1)$, teenage aspiration $(F 2)$, and occupational attainment $(F 3)$. The sample consists of 6,407 cases from the 1970 British Cohort Study (BCS70). Table 7 summarizes the sample covariance matrix of the variables.
Stage 1 Figure 4a shows the original model, M1. Our aim is to compare the standardized coefficients of educational achievement $\left(\gamma_{1}{ }^{*}\right)$ and teenage aspiration $\left(\gamma_{2}{ }^{*}\right)$ on occupational attainment. The model is fitted to the observed data by using EQS. The chi-square goodness-of-fit statistics are $\chi^{2}=1.199, d f=6, p>.05$.

We follow the general framework to transform M1 into the standardized model, M2. Fig. 4b depicts the standardized model, M2. Since the latent factors $F 1, F 2$, and $F 3$ are the target factors that we are going to standardize, each latent factor is regressed on a DLV (F4 to $F 6$ ). Variance of $F 4$ and $F 5$ are fixed at 1.0. F7 is the disturbance term of $F 6$. 
Table 5 Summary of the standardized parameter estimates and their estimated standard error in Example 2 by analysis using different structural equation modeling programs and approaches

\begin{tabular}{|c|c|c|c|c|c|c|c|c|c|c|}
\hline \multirow[t]{3}{*}{ Program method parameter } & \multicolumn{6}{|c|}{ EQS6.1 } & \multirow{2}{*}{\multicolumn{2}{|c|}{$\frac{\text { Mplus5.2 }}{\text { Built-in }^{\text {b }}}$}} & \multirow{2}{*}{\multicolumn{2}{|c|}{$\frac{\text { LISREL8.8 }}{\text { Phantom variable }}$}} \\
\hline & \multicolumn{2}{|c|}{ Reparameterization } & \multicolumn{2}{|c|}{ Correlation } & \multicolumn{2}{|c|}{ Bootstrap } & & & & \\
\hline & Est. & $S E$ & Est. & $S E$ & Est. & $S E$ & Est. & $S E$ & Est. & $S E$ \\
\hline$\gamma_{1}^{*}$ & -.245 & .081 & -.245 & .082 & -.242 & .075 & -.245 & .081 & -.245 & .082 \\
\hline$\gamma_{2}{ }^{*}$ & .442 & .079 & .442 & .083 & .440 & .082 & .442 & .079 & .442 & .080 \\
\hline$\gamma_{3}^{*}$ & .310 & .064 & .310 & .067 & .306 & .065 & .310 & .064 & .310 & .065 \\
\hline$\beta^{*}$ & .239 & .067 & .239 & .069 & .238 & .066 & .239 & .067 & .239 & .067 \\
\hline$\phi_{12}{ }^{*}$ & .661 & .040 & .661 & .085 & .660 & .038 & .661 & .040 & .661 & .040 \\
\hline$\phi_{13}{ }^{*}$ & .355 & .062 & .355 & .075 & .353 & .057 & .355 & .062 & .355 & .062 \\
\hline$\phi_{23} *$ & .374 & .061 & .374 & .076 & .377 & .057 & .374 & .061 & .374 & .061 \\
\hline
\end{tabular}

Est. = parameter estimate; $S E=$ standard error; standard errors that deviate substantially from estimates of other methods are in bold face.

${ }^{a}$ Regular (i.e., completely nonparametric) bootstrap is used; replication $=1,000$

${ }^{\mathrm{b}}$ The StdYX standardization are reported here. StdYX uses the variances of the continuous latent variables, as well as the variances of the background and outcome variables for standardization (L. K. Muthèn \& B. O. Muthèn, 2007)

$F 999$ is the phantom variable with unit variance, and the path from $F 999$ to $F 7$ is fixed at 1.0. $F 8=F 4^{\prime}$ and $F 9=F 5^{\prime}$ are image latent variables with negative unit variance, and they form the image structure of $F 4$ and $F 5$.

M2 is then fitted to the observed data. The path coefficients of the image latent variables, $F 8$ and $F 9$ are constrained to be equal to the coefficients of the corresponding target variables $F 4$ and $F 5$. Covariance between $F 8$ and $F 9$ is constrained to be equal to the negative of the covariance between $F 4$ and $F 5$. Three linear constraints are imposed on the model altogether. A comparison with the model chi-square of M1, M2 gives the same chi-square value, $\chi^{2}=1.199, d f=6, p>.05$, indicating that the two models are covariance equivalent and have the same implied covariance matrix. Table 8 shows the parameter estimates and their $S E$ s. The parameter

Table 6 Hypothesis testing results based on the unstandardized model (M1) and standardized model (M2) in Example 2

\begin{tabular}{|c|c|c|c|c|c|c|c|c|}
\hline \multirow{3}{*}{$\begin{array}{l}\text { Model } \\
\text { Parameter }\end{array}$} & \multicolumn{4}{|c|}{ M1 (Unstandardized) } & \multicolumn{4}{|c|}{ M2 (Standardized) } \\
\hline & \multicolumn{2}{|c|}{ Unconstrained } & \multicolumn{2}{|c|}{ Constrained $^{\mathrm{a}}$} & \multicolumn{2}{|c|}{ Unconstrained } & \multicolumn{2}{|c|}{ Constrained $^{\mathrm{b}}$} \\
\hline & Est. & $S E$ & Est. & $S E$ & Est. & $S E$ & Est. & $S E$ \\
\hline$\gamma_{1} / \gamma_{1}^{*}$ & -.014 & .005 & .011 & .003 & -.245 & .081 & .164 & .024 \\
\hline$\gamma_{2} / \gamma_{2} *$ & .124 & .023 & .011 & .003 & .442 & .079 & .164 & .024 \\
\hline$\gamma_{3} / \gamma_{3} *$ & .285 & .061 & .011 & .003 & .310 & .064 & .164 & .024 \\
\hline Model chi-square $\left(\chi^{2}\right)$ & \multicolumn{2}{|l|}{13.218} & \multicolumn{2}{|l|}{62.244} & \multicolumn{2}{|l|}{13.218} & \multicolumn{2}{|l|}{39.097} \\
\hline \multirow[t]{2}{*}{ Degree of freedom $(d f)$} & 3 & & 5 & & 3 & & 5 & \\
\hline & \multicolumn{8}{|c|}{ Likelihood ratio test results } \\
\hline Hypothesis & $\Delta \chi^{2}$ & $\Delta d f$ & & $p$ & $\Delta \chi^{2}$ & $\Delta d f$ & & $p$ \\
\hline \multirow[t]{2}{*}{$\mathrm{H}_{0}: \gamma_{1}=\gamma_{2}=\gamma_{3} / \mathrm{H}_{0}: \gamma_{1}^{*}=\gamma_{2} *=\gamma_{3}^{*}$} & 49.026 & 2 & & $<.001$ & 25.879 & 2 & & $<.001$ \\
\hline & \multicolumn{8}{|c|}{ Lagrange multiplier test (for releasing constraints) results } \\
\hline Hypothesis & $\chi^{2}$ & $d f$ & & $p$ & $\chi^{2}$ & $d f$ & & $p$ \\
\hline $\mathrm{H}_{0}: \gamma_{1}=\gamma_{3} / \mathrm{H}_{0}: \gamma_{1} *=\gamma_{3}^{*}$ & $39.252 *$ & 1 & & $<.001$ & $22.999 *$ & 1 & & $<.001$ \\
\hline $\mathrm{H}_{0}: \gamma_{2}=\gamma_{3} / \mathrm{H}_{0}: \gamma_{2} *=\gamma_{3} *$ & $25.404 *$ & 1 & & $<.001$ & 3.403 & 1 & & .065 \\
\hline
\end{tabular}

Est. = parameter estimate; $S E=$ standard error.

${ }^{*}$ Result is significant at 5\% significance level

${ }^{\text {a }}$ Constrained model is fitted under $\mathrm{H}_{0}: \gamma_{1}=\gamma_{2}=\gamma_{3}$

${ }^{\mathrm{b}}$ Constrained model is fitted under $\mathrm{H}_{0}: \gamma_{1} *=\gamma_{2} *=\gamma_{3}$ * 
Table 7 Covariance matrix for the 1970 British cohort $(N=6407)$ from Schoon and Parson's $(2002)$ study

\begin{tabular}{|c|c|c|c|c|c|c|}
\hline & Exam score $(V 1)$ & Highest qualifications $(V 2)$ & Job aspiration $(V 3)$ & Educational aspiration ( V4) & Goldthorpe (V5) & RGSC (V6) \\
\hline$V 1$ & 263.413 & & & & & \\
\hline$V 2$ & 5.996 & 0.436 & & & & \\
\hline$V 3$ & 2.312 & 0.067 & 0.212 & & & \\
\hline$V 4$ & 7.591 & 0.213 & 0.164 & 0.722 & & \\
\hline$V 5$ & 78.740 & 2.271 & 1.295 & 4.125 & 244.923 & \\
\hline$V 6$ & 6.622 & 0.190 & 0.110 & 0.347 & 14.648 & 1.440 \\
\hline
\end{tabular}

estimates and their $S E$ s are the same as the standardized parameter estimates and their corresponding $S E$ s reported by Mplus, suggesting that we successfully transformed the model into the standardized model.

Stage 2 To test the equality of the two standardized coefficients from $F 1$ and $F 2$ on $F 3$, we fit a constrained model by imposing a linear equality constraint - that is, $F 4 \rightarrow F 6=F 5 \rightarrow F 6-$ on M2. Table 8 shows the model chisquares and the parameter estimates of the constrained and unconstrained model (under the heading "Standardized"). We compare the chi-square of the constrained model with that of the unconstrained model. The LR test gives $\Delta \chi^{2}=$ $2.981-1.199=1.782, \Delta d f=7-6=1, p>.05$, suggesting that the relative effects of educational achievement and teenage aspiration on occupational attainment are not significantly different from each other. As compared with the Wald test results based on the analysis by Mplus, the two tests give the same conclusion about the two coefficients.

\section{Discussion}

In this article, a method for comparing standardized coefficients in SEM is proposed. Since different variables are often measured in different units in behavioral research, comparing their standardized effects will lead to a more meaningful conclusion, because they are affected less by the units of measurement. Three real examples are given to demonstrate the implementation of the proposed method. In all the examples, our method performs accurately in standardizing the model at stage 1 . It provides the same standardized parameter estimates and standard errors, as compared with those reported by Mplus and LISREL (using the phantom variables approach). At stage 2, the LR test can be employed as a routine step to compare the coefficients of interest.

We also compare the LR test with the Wald test results in the regression example at the beginning of this article and
Example 3. Theoretically, these tests address the same question, and they are asymptotically equivalent under the same null hypothesis (Chou \& Bentler, 1990; Satorra, 1989). From Tables 2 and 8 , the $p$-values reported by the LR test and the Wald test are highly comparable, suggesting that the LR test at stage 2 behaves similarly to the Wald test used by the phantom variable approach for comparing standardized coefficients.

It is worthwhile noting that the comparison of coefficients based on the unstandardized and standardized metrics lead to different statistical conclusions throughout our examples (see Tables 2, 4, 6, and 8), because the two tests test different null hypotheses, as was discussed previously. If researchers fail to recognize the difference between the two tests and make inference about one metric on the basis of the analysis of the other, they may risk drawing a misleading conclusion. Researchers should pay special attention to the difference between the two metrics, especially when the variances of the variables are very different, and should choose the appropriate metric for testing according to the questions they are going to address.

There are several distinguishing features of the proposed method. First, it gives accurate standard error estimates for the standardized parameters. To obtain the standardized estimates, it is procedurally tempting for one to standardize the variables first and analyze the data on the basis of the correlation matrix, because this can save lots of effort. However, as is shown in Table 3 and 5 (under the heading of "Correlation"), an analysis based on the correlation matrix in general gives us correct parameter estimates but incorrect SEs (e.g., Bentler, 2007; Cheung, 2009a; Cudeck, 1989). When we analyze the correlation matrix, the variances of the dependent variables depend on other random parameters and are, therefore, subject to sampling variability (i.e., they are not fixed at 1.0 nonstochastically), which eventually affects the accuracy of the standard error estimates. We can further verify this by comparing the correlation-based $S E$ s and our $S E$ estimates with the bootstrap $S E$ s. Tables 3 and 5 (under the heading "Bootstrap") show the standardized parameter estimates 
Fig. 4 Structural model in

Example 3. a Original model

(M1). b Standardized model

(M2). $F 1=$ educational

achievement, $F 2$ = teenage

aspiration, $F 3=$ occupational

attainment. Labels for parame-

ters of interest are printed (a)

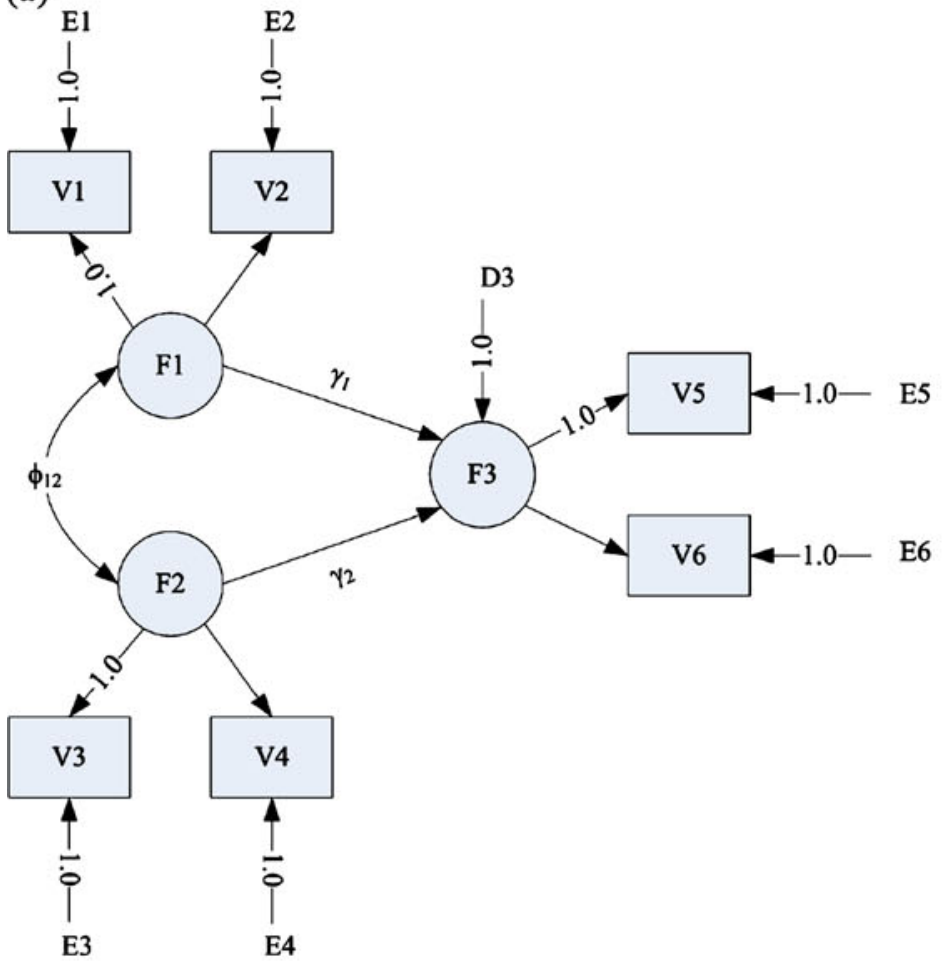

(b)

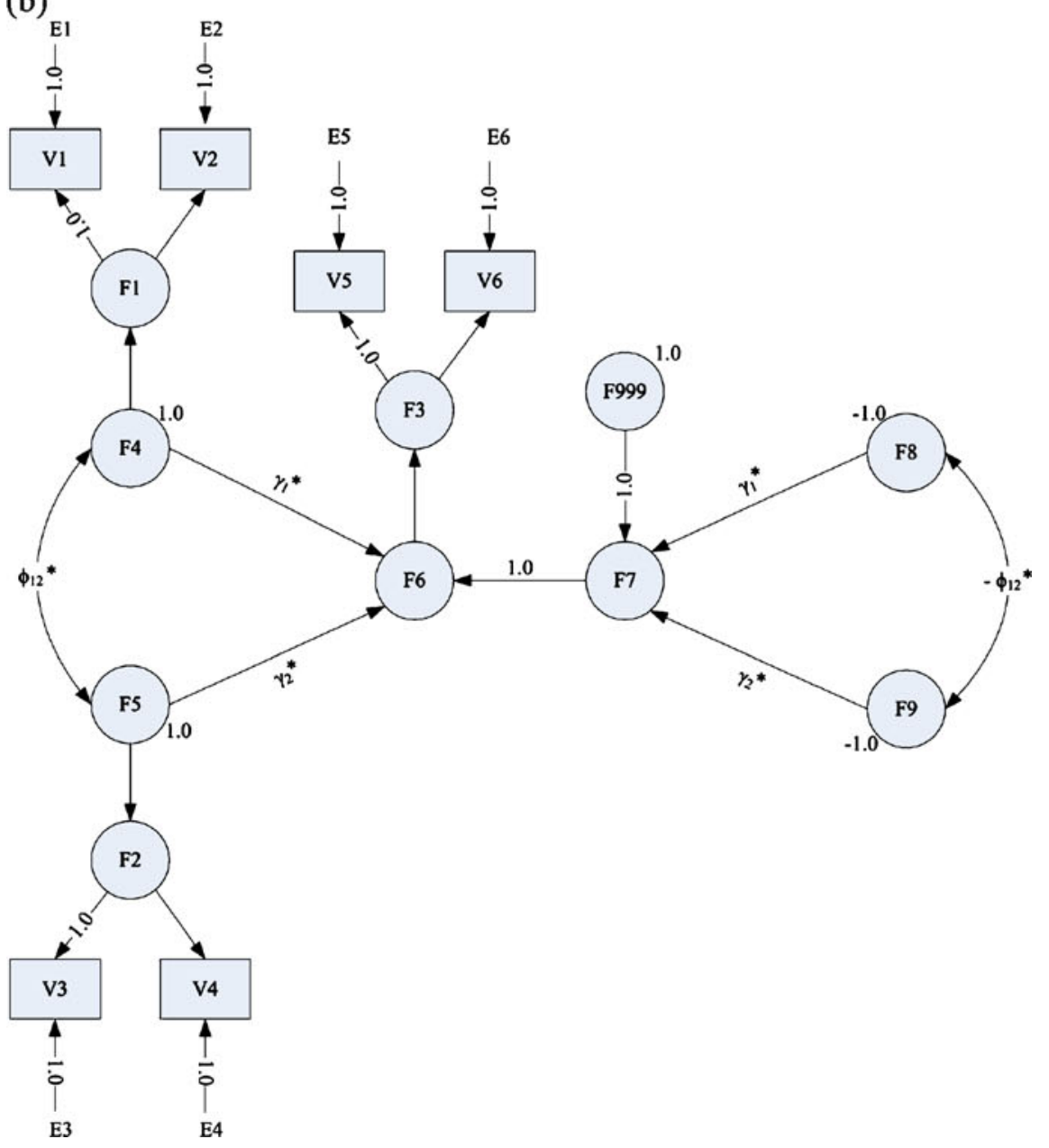


Table 8 Summary of the parameter estimates and hypothesis test results in Example 3 by analysis using EQS and Mplus

\begin{tabular}{|c|c|c|c|c|c|c|c|c|c|c|c|c|}
\hline \multirow{4}{*}{$\begin{array}{l}\text { Program metrics } \\
\text { model Fitted } \\
\text { Parameters }\end{array}$} & \multicolumn{8}{|l|}{ EQS } & \multicolumn{4}{|l|}{ Mplus } \\
\hline & \multicolumn{4}{|c|}{ Unstandardized } & \multicolumn{4}{|c|}{ Standardized } & \multicolumn{2}{|c|}{ Unstandardized } & \multicolumn{2}{|c|}{ Standardized $^{\mathrm{a}}$} \\
\hline & \multicolumn{2}{|c|}{$\begin{array}{l}\text { Unconstrained } \\
\text { M1 }\end{array}$} & \multicolumn{2}{|c|}{$\begin{array}{l}\text { Constrained } \\
\text { M1 }\end{array}$} & \multicolumn{2}{|c|}{$\begin{array}{l}\text { Unconstrained } \\
\text { M2 }\end{array}$} & \multicolumn{2}{|c|}{$\begin{array}{l}\text { Constrained } \\
\text { M2 }\end{array}$} & \multicolumn{2}{|c|}{ Unconstrained M1 } & \multicolumn{2}{|c|}{ Unconstrained M1 } \\
\hline & Est. & $S E$ & Est. & $S E$ & Est. & $S E$ & Est. & $S E$ & Est. & $S E$ & Est. & $S E$ \\
\hline$\gamma_{1} / \gamma_{1} *$ & 0.192 & 0.024 & 0.418 & 0.015 & 0.212 & 0.025 & 0.245 & 0.006 & 0.192 & 0.024 & 0.212 & 0.025 \\
\hline$\gamma_{2} / \gamma_{2} *$ & 16.333 & 1.570 & 0.418 & 0.015 & 0.278 & 0.026 & 0.245 & 0.006 & 16.333 & 1.570 & 0.278 & 0.026 \\
\hline$\phi_{12} / \phi_{12}{ }^{*}$ & 2.332 & 0.095 & 2.341 & 0.096 & 0.713 & 0.014 & 0.710 & 0.014 & 2.332 & 0.095 & 0.713 & 0.014 \\
\hline $\begin{array}{l}\text { Model } \\
\text { chi-square }\end{array}$ & \multicolumn{4}{|c|}{$\begin{array}{cc}\chi^{2}=1.199, & \chi^{2}=112.524, \\
d f=6, p>.05 & d f=7, \\
& p>.001 \\
\text { Testing } \mathrm{H}_{0}: \gamma_{1}=\gamma_{2} / \mathrm{H}_{0}: \gamma_{1}^{*}=\gamma_{2}^{*}\end{array}$} & \multicolumn{2}{|c|}{$\begin{array}{l}\chi^{2}=1.199 \\
d f=6 \\
p>.05\end{array}$} & \multicolumn{2}{|c|}{$\begin{array}{l}\chi^{2}=2.981 \\
d f=7 \\
p>.05\end{array}$} & \multicolumn{2}{|c|}{$\begin{array}{l}\chi^{2}=1.199 \\
\quad d f=6, p>.05\end{array}$} & \multicolumn{2}{|c|}{$\begin{aligned} \chi^{2} & =1.199, d f=6 \\
p & >.05\end{aligned}$} \\
\hline $\begin{array}{l}\text { Likelihood } \\
\text { ratio test }\end{array}$ & \multicolumn{4}{|c|}{$\Delta \chi^{2}=111.325, \Delta d f=1, p<.001$} & \multicolumn{4}{|c|}{$\Delta \chi^{2}=1.782, \Delta d f=1, p=.182$} & \multicolumn{2}{|l|}{-} & \multicolumn{2}{|l|}{-} \\
\hline Wald test ${ }^{\mathrm{d}}$ & \multicolumn{4}{|l|}{-} & \multicolumn{4}{|l|}{-} & \multicolumn{2}{|c|}{$\begin{array}{l}\widehat{\gamma}_{2}-\widehat{\gamma}_{1}=16.141 \\
\quad S E=1.588 \\
\left(\widehat{\gamma}_{2}-\widehat{\gamma}_{1}\right) / S E=10.162 \\
p<.001\end{array}$} & \multicolumn{2}{|c|}{$\begin{array}{l}\widehat{\gamma}_{2}^{*}-\widehat{\gamma}_{1}^{*}=0.066 \\
S E=0.050 \\
\left(\widehat{\gamma}_{2}^{*}-\widehat{\gamma}_{1}^{*}\right) / S E= \\
1.325, p=.185\end{array}$} \\
\hline
\end{tabular}

Est. = parameter estimate; $S E=$ standard error

${ }^{a}$ The StdYX standardization is reported here. StdYX uses the variances of the continuous latent variables, as well as the variances of the background and outcome variables for standardization (L. K. Muthèn \& B. O. Muthèn, 2007)

$\mathrm{b}$ The constrained model is fitted under $\mathrm{H}_{0}: \gamma_{1}=\gamma_{2}$

${ }^{\mathrm{c}}$ The constrained model is fitted under $\mathrm{H}_{0}: \gamma_{1} *=\gamma_{2} *$

${ }^{\mathrm{d}}$ Wald test resultsare based on the analysis by using Mplus5.2

and their corresponding SEs by bootstrapping. ${ }^{10}$ As was expected, the correlation-based $S E$ s deviate quite substantially from the bootstrap estimates for some of the parameters, suggesting that they are problematic. In contrast, the $S E$ estimates given by our method agree with the bootstrap $S E$ s up to two decimal places, suggesting that they can be trusted generally. Hence, as is shown in our Examples 1 and 2, it is generally inappropriate to compare the standardized coefficients based on the analysis of correlations unless the method of constrained estimation, which gives the correct $S E$ s, is implemented when a correlation matrix is analyzed (see Browne, 1982; Browne \& Mels, 1992; Mels, 1989).

Second, the method is compatible with all major SEM software programs on the market. Unlike other approaches, our method does not involve the use of nonlinear constraints, and it requires only the basic standard functions to work. Although we demonstrate the implementation of the method only by using EQS, the method can work well

\footnotetext{
${ }^{10}$ The application of bootstrapping in SEM has been discussed extensively (e.g., Bollen \& Stine, 1993; Yung \& Bentler, 1996). The bootstrap method does not rely on any distributional assumption about the data. Therefore, it is expected to outperform the traditional method for giving more accurate $S E$ estimates when theoretical assumptions underlying tradition parametric statistical inferences are in doubt.
}

with other programs, such as AMOS, ${ }^{11}$ too. Researchers can choose their favorite SEM programs for implementing the proposed method.

In relation to this, another advantage of our method is that one can keep the use of programming to a minimum. The phantom variable approach requires researchers to fully understand the functional relationships ${ }^{12}$ among the model parameters before they can specify the nonlinear constraints on the additional parameters correctly. In contrast, one can use the graphical programming capabilities possessed by some SEM software programs (e.g., EQS and AMOS) to implement the proposed method and avoid the complicated

\footnotetext{
${ }^{11}$ AMOS users cannot follow the proposed method to impose constraints on the covariances (i.e. $\operatorname{cov}\left(F_{\mathrm{i}}{ }^{\prime}, F_{\mathrm{i}}{ }^{\prime}\right)=-\operatorname{cov}\left(F_{\mathrm{i}}, F_{\mathrm{i}}\right)$, because AMOS does not support the specification of constraints, which are in the form of $a=-b$. Interested readers may find a solution for this limitation, see Electronic Supplementary Material.

${ }^{12}$ For example, one must understand the functional relationship of $V 4$ with $V 1, V 2$, and $V 3$ in Example 1 before he/she knows to specify the variance of $V 4$ as $\left(\gamma_{1}{ }^{2} \operatorname{var}(V 1)+\gamma_{2}{ }^{2} \operatorname{var}(V 2)+\gamma_{3}{ }^{2} \operatorname{var}(V 3)+\right.$ $2 \gamma_{1} \gamma_{2} \operatorname{cov}(V 1, V 2)+2 \gamma_{1} \gamma_{3} \operatorname{cov}(V 1, V 3)+2 \gamma_{2} \gamma_{3} \operatorname{cov}(V 2, V 3)+$ var $(E 4))$ and, hence, the difference between two standardized coefficients $\left(\gamma_{1}{ }^{*}-\gamma_{2}{ }^{*}\right)$ as $\left(\gamma_{1}(\operatorname{var}(V 1))^{\wedge} 0.5-\gamma_{2}(\operatorname{var}(V 2))^{\wedge} 0.5\right) /$ $\left(\gamma_{1}^{2} \operatorname{var}(V 1)+\gamma_{2}^{2} \operatorname{var}(V 2)+\gamma_{3}{ }^{2} \operatorname{var}(V 3)+2 \gamma_{1} \gamma_{2} \operatorname{cov}(V 1, V 2)+\right.$ $\left.2 \gamma_{1} \gamma_{3} \operatorname{cov}(V 1, V 3)+2 \gamma_{2} \gamma_{3} \operatorname{cov}(V 2, V 3)+\operatorname{var}(E 4)\right)^{\wedge} 0.5$. We believe this task is difficult and tedious for many applied researchers.
} 
syntax. Indeed, many SEM beginners welcome SEM software programs such as EQS and AMOS because of their well-designed graphical user interface (e.g., Kline, 1998). Some SEM practitioners may find our proposed method favorable because they can follow the general framework of model transformation and use the graphical interface to specify the standardized model without going into the mathematical details.

Finally, the proposed method is capable of comparing three or more standardized coefficients simultaneously. By using the LR test at stage 2, we can test the equality of $k$ standardized coefficients by simply imposing $(k-1)$ linear equality constraints on the standardized coefficients and comparing the chi-square statistics between the constrained and unconstrained standardized models. As a posthoc comparison, we can apply the Lagrange multiplier (LM) tests to further evaluate the significance of each equality constraint in a pairwise fashion after an overall significant LR test result has been observed. For example, we can further test the null hypotheses $\mathrm{H}_{0}: \gamma_{1}{ }^{*}=\gamma_{3}{ }^{*}$ and $\mathrm{H}_{0}: \gamma_{2}{ }^{*}=$ $\gamma_{3}{ }^{*}$ after finding an overall significant difference among the three standardized coefficients by using LM test in Example 2 (see Table 6). The LM test results show that releasing the equality constraint, $\gamma_{1}{ }^{*}=\gamma_{3}{ }^{*}$, yields a significant improvement in model fit for the standardized model with $\chi^{2}=$ 23.00, $d f=1, p<.001$.This improvement means that there is a significant difference between the standardized coefficients, $\gamma_{1}^{*}$ and $\gamma_{3}{ }^{*}$. In contrast, releasing the equality constraint, $\gamma_{2}{ }^{*}=\gamma_{3}{ }^{*}$, in the standardized model does not significantly improve the model fit, with $\chi^{2}=3.40, d f=1$, $p>.05$, suggesting that there is no significant difference between the standardized coefficients, $\gamma_{2}{ }^{*}$ and $\gamma_{3}{ }^{*}$.

\section{Conclusion}

For many years, methodologists have studied how different parametric statistical procedures such as canonical correlation analysis can be incorporated into SEM (e.g., Fan, 1997; Graham, 2008). One reason is that many of these multivariate techniques do not provide the $S E$ s for different types of coefficients and, therefore, statistical significance tests cannot be conducted. The present study shows that if these coefficients are made explicit in the model (i.e., appear as a model parameter in the specified model), we can easily obtain the $S E$ and carry out subsequent testing involving these coefficients by using standard SEM analysis. Our proposed method demonstrates one of the usages of the model reparameterization technique in this area of study.

In this article, only limited kinds of models are considered in order to demonstrate how the method can be applied for making relevant statistical inference. Model transformation will become more tedious if the original model is complex. However, the general principle of model transformation for more complex models remains unchanged. Although more image latent variables and a larger image structure are involved for a more complex model, it does not influence the effectiveness of the proposed method for comparing standardized coefficients. Nevertheless, the proposed model may fail in other extreme model conditions, such as a nonrecursive model with the presence of reciprocal effects. Further investigation is required to evaluate the effectiveness of the proposed method in these conditions.

Future studies can also be done to explore how model reparameterization can be used for testing other parameters, such as standardized indirect effects and squared multiple correlation coefficients $\left(R^{2}\right)$ (Kwan \& Chan, 2010). Considering the general model in Fig. $2, R^{2}$ can be defined as the proportion of total variance of $Y$ that is accounted for by the predictors $(X 1 \ldots X k)$. Mathematically, it can be expressed as

$R^{2}=1-\operatorname{var}(D)=1-[1-g(\theta)]=g(\theta)$

Therefore, $R^{2}$ is, in fact, equivalent to $g(\theta)$, which is defined as the total variances and covariances due to the antecedent variables in the standardized model. It will be interesting to reparameterize $g(\theta)$ as a single model parameter in the transformed model, so that we can conduct significance tests and subsequent analysis involving $R^{2}$.

Finally, in addition to raising researchers' awareness about the difference between the tests of coefficients based on standardized and unstandardized metrics, we hope that our proposed method can also inspire methodologists about the potential usefulness of model reparameterization as a general modeling technique in SEM. Future research can probably be done to explore how model reparameterization can be a useful technique in other kinds of SEM analysis.

\section{References}

Arbuckle, J. L. (2007). AMOS 16.0 user's guide. Chicago: SPSS.

Bentler, P. M. (1995). EQS structural equations program manual. Encino: Multivariate Software.

Bentler, P. M. (2007). Can scientifically useful hypotheses be tested with correlations? The American Psychologist, 62, 772-782.

Bollen, K. A., \& Stine, R. (1993). Bootstrapping goodness-of-fit measures in structural equation models. In K. A. Bollen \& J. S. Long (Eds.), Testing structural equation models (pp. 111-135). Newbury Park: Sage.

Browne, M. W. (1982). Covariance structures. In D. M. Hawkins (Ed.), Topics in applied multivariate analysis. Cambridge: Cambridge University Press.

Browne, M. W., \& Mels, G. (1992). RAMONA user's guide. Columbus: Ohio State University, Department of Psychology. 
Chan, W. (2007). Comparing indirect effects in structural equation modeling: A sequential model fitting method using covarianceequivalent specifications. Structural Equation Modeling, 14, 326-346.

Cheung, M. W. L. (2009a). Comparison of methods for constructing confidence intervals of standardized indirect effects. Behavior Research Methods, 41, 425-438.

Cheung, M. W. L. (2009b). Constructing approximate confidence intervals for parameters with structural equation models. Structural Equation Modeling, 16, 267-294.

Chou, C. P., \& Bentler, P. M. (1990). Model modification in covariance structure modeling: A comparison among likelihood ratio, Lagrangemultiplier, and Wald tests. Multivariate Behavioral Research, 25, 115-136.

Cudeck, R. (1989). Analysis of correlation matrices using covariance structure models. Psychological Bulletin, 105, 317-327.

Fan, X. (1997). Canonical correlation analysis and structural equation modeling: What do they have in common? Structural Equation Modeling, 4, 65-79.

Fox, J. (1997). Applied regression analysis, linear modes, and related methods. Thousand Oaks: Sage.

Graham, J. M. (2008). The general linear model as structural equation modeling. Journal of Educational and Behavioral Statistics, 33, 485-506.

Guo, B., Perron, B. E., \& Gillespie, D. F. (2009). A systematic review of structural equation modeling in social work research. British Journal of Social Work, 39, 1556-1574.

Hershberger, S. L. (2003). The growth of structural equation modeling: 1994-2001. Structural Equation Modeling, 3, 93-104.

Jackson, D. L., Gillaspy, J. A., Jr., \& Purc-Stephenson, R. (2009). Reporting practices in confirmatory factor analysis: An overview and some recommendations. Psychological Methods, $14,6-23$.

Jöreskog, K. G., \& Sörbom, D. (1996). LISREL 8: A user's reference guide. Chicago: Scientific Software International.
Kline, R. B. (1998). Software review: Software programs for structural equation modeling: Amos, EQS, and LISREL. Journal of Psychoeducational Assessment, 16, 343-364.

Kwan, J. L.Y., \& Chan, W. (2010). Linearization of nonlinear parameters in structural equation modeling: A model reparameterization approach. Poster presented at the Association for Psychological Science 22nd Annual Convention, Boston.

Loehlin, J. C. (2004). Latent variable models: An introduction to factor, path, and structural equation analysis. Mahwah: Erlbaum.

Mels, G. (1989). A general system for path analysis with latent variables. Unpublished master's thesis, University of South Africa, Department of Statistics.

Muthén, L. K., \& Muthén, B. O. (2007). Mplus user's guide (5th ed.). Los Angeles: Muthén \& Muthén.

Organization for Economic Cooperation and Development. (2009). PISA data analysis manual: SPSS (2nd ed.). Paris: Organization for Economic Cooperation and Development.

Rindskopf, D. (1984). Using phantom and imaginary latent variables to parameterize constraints in linear structural models. Psychometrika, 49, 37-47.

Satorra, A. (1989). Alternative test criteria in covariance structure analysis: A unified approach. Psychometrika, 54, 131-151.

Schoon, I., \& Parsons, S. (2002). Teenage aspirations for future careers and occupational outcomes. Journal of Vocational Behavior, 30, 262-288.

Steiger, J. H. (1995). SEPATH module. In Statistica for Windows, computer software manual. Tulsa, OK: StatSoft, Inc.

Tremblay, P. F., \& Gardner, R. C. (1996). On the growth of structural equation modeling in psychological journals. Structural Equation Modeling, 3, 93-104.

Yung, Y., \& Bentler, P. M. (1996). Bootstrapping techniques in analysis of mean and covariance structures. In G. A. Marcoulides \& R. E. Schumacker (Eds.), Advanced structural equation modeling: Issues and techniques (pp. 195-226). Mahwah: Erlbaum. 